doi: $10.2306 /$ scienceasia1513-1874.2014.40.219

\title{
Residual lung volume of female Thai adults
}

\author{
Arpalak Paksaichol $^{\mathrm{a}}$, Ganyapong Chaturapanich ${ }^{\mathrm{a}}$, Chulaluk Komoltri $^{\mathrm{b}}$, Ratchakrit Srikuea ${ }^{\mathrm{a}}$, \\ Chumpol Pholpramool ${ }^{\mathrm{a}, *}$ \\ a Department of Physiology, Faculty of Science, Mahidol University, Bangkok 10400 Thailand \\ b Department of Research and Development, Faculty of Medicine, Siriraj Hospital, Mahidol University, \\ Bangkok 10700 Thailand
}

*Corresponding author, e-mail: chumpol.pho@mahidol.ac.th

Received 13 Jan 2014

Accepted 18 May 2014

\begin{abstract}
Lung volumes including residual volume (RV) are ethnicity-dependent parameters which are useful in clinical diagnosis of lung diseases and in assessing body density, and hence total body adiposity. The latter predisposes an individual to cardiovascular and/or metabolic diseases which are serious health problems worldwide. At present, RV of Thai people has not been reported. This study determines RV of young adult Thai women and proposes a prediction equation to compare it with other equations previously reported for the Asian population. A group of 161 healthy young women aged between 18 and 29 years were recruited for constructing and cross-validating the equation. RV was measured by an $\mathrm{O}_{2}$ dilution technique using a computerized spectrometer. Anthropometric variables were determined using a beam balance, a stadiometer, and bioelectric impedance analysis. The mean residual volume for the selected group of young Thai women is $1.18 \pm 0.23 \mathrm{l}$ and best described by the equation $\mathrm{RV}=-3.236+0.024 H+0.028 A$ where $H$ is the height in $\mathrm{cm}$ and $A$ is the age in years. Goldman and Becklake's equation for Caucasians overestimated our RV whereas those of Ching and Horsfall, and Demura et al for Chinese and Japanese people, respectively, underestimated it. Chin's equation for Singaporeans yields similar value to the measured residual volume. The results confirm that residual volume is ethnically and geographically dependent. The equations for Thais and Singaporean of the same age and sex are comparable.
\end{abstract}

KEYWORDS: bioelectric impedance analysis, body composition, body fat, body density, prediction equation

\section{INTRODUCTION}

Body composition is an essential component in the description of physical characteristics of an individual. There are several methods available for the assessment of body composition in field work and in laboratories such as skinfold thickness, bioelectric impedance analysis (BIA), densitometry, dual energy X-ray absorptiometry, and magnetic resonance imaging. BIA is suitable for the measurements in field studies since it is portable and requires less technical skill. It is also a common method in fitness clubs or health spas. On the other hand, densitometry is a laboratory method that requires more technical skill and subject cooperation during the measurement. Densitometry is nevertheless accepted as a 'criterion method' used to validate other assessments ${ }^{1}$. To determine body density, the body weight of a subject is measured in air and in water with or without a direct measurement of residual lung volume (RV), while gastrointestinal volume is assumed to be $100 \mathrm{ml}$. To assess RV, a special instrument such as a computerized spectrometer or a whole body plethysmograph, not normally available in clinics, health clubs or fitness centres, is required. A prediction equation for $\mathrm{RV}$ is therefore useful when direct measurement cannot be made.

It is well documented that, in addition to sex, age, weight, and height, ethnic background of healthy individuals contributes to variance of both volumes and functions of the lung ${ }^{2-6}$. Furthermore, previous studies have shown that the RV of Asian populations are different depending on their ancestry, i.e., Chinese, Malay, or Indian Singaporean ${ }^{7}$, and geography, i.e., Cantonese, Singaporean Chinese, or Taiwanese ${ }^{8-10}$. Thus a prediction equation is valid only for a specific population. At present, a prediction equation for RV in Thai population is still unavailable. Herein we measured RV of young adult women using a gaswashout technique with a computerized spectrometer and proposed a prediction equation for the RV of Thai women.

\section{METHODS}

\section{Participants}

One hundred and sixty-one women aged between 18 and 29 years, who lived in Bangkok, Thailand, were recruited in this study. They were randomly divided, using computer generated random numbers, into two groups: a validation group of 121 women to 
construct prediction equations and a cross-validation group of 40 women for testing external validity of the proposed equations. The number of participants of the validation was three times that of the cross-validation group to get enough sample size for construction of a valid regression equation.

All participants were in good health based on their medical history and physical examinations. They neither smoke nor took any medicine during the period of the study. Informed consents were signed by all participants and the study protocol was approved by Mahidol University Institutional Review Board (protocol 281/1999, approved on February 12th, 1999). The research was conducted in accordance with the principles set forth in the Helsinki Declaration.

\section{Anthropometric measurements}

Body weights were measured using a beam balance (Weylux model 424 J, E.H. Oakley \& Co Ltd, Aylesbury, UK) to within $0.1 \mathrm{~kg}$. Standing heights were measured with a stadiometer to within $0.1 \mathrm{~cm}$ at full inspiration of the participants ${ }^{11}$. Body fat (BF) was obtained after subtraction of fat-free mass (FFM), which was assessed by bioelectric impedance analysis using BIA-101 impedance analyser (RJL system, Detroit, MI, USA), from body weight.

\section{Measurement of residual lung volume}

Residual volume of lung (RV) was measured by $\mathrm{O}_{2}$ dilution technique using a computerized spectrometer (Sensomedic Vmax 299, SenSormedic Corp, Yorba Linda, CA, USA). Participants wore light-weight dresses to allow free chest movement and sat in an arm chair in an erect position. They breathe normally through a mouth piece with a nose clip. The test started by asking the participants to fully inspire and completely expire, then breathe normally. All participants were allowed to become familiarized with the procedures before testing. Three measurements were made and the minimal RV was recorded. All spirometry measurements and recording followed the American Thoracic Society and the European Respiratory Society ${ }^{12}$.

\section{Statistical analysis}

Data are presented as mean \pm SD. Pearson correlation was used to test the relationship of measured RV (mRV) with height, age, and weight. Linear regression were applied to evaluate the relationship of $m R V$ with the independent variables: height, age, and weight. A paired $t$-test was used to test the difference between $\mathrm{mRV}$ and predicted RV (pRV). A scatter plot of $\mathrm{mRV}$ and $\mathrm{pRV}$ was used to gauge agreement
Table 1 Physical characteristics of participants.

\begin{tabular}{lrcc}
\hline Characteristic & Validation & Cross-validation & $p$-value \\
\hline age $($ years $)$ & $23 \pm 3$ & $24 \pm 3$ & 0.448 \\
Height $(\mathrm{cm})$ & $156 \pm 5$ & $154 \pm 5$ & 0.082 \\
Weight $(\mathrm{kg})$ & $53 \pm 9$ & $50 \pm 9$ & 0.190 \\
BMI $\left(\mathrm{kg} / \mathrm{m}^{2}\right)$ & $22 \pm 3$ & $21 \pm 3$ & 0.454 \\
BF $(\%)$ & $25 \pm 5$ & $24 \pm 4$ & 0.409 \\
FFM $(\mathrm{kg})$ & $39 \pm 5$ & $28 \pm 5$ & 0.167
\end{tabular}

$\mathrm{BMI}=$ body mass index BF $=$ body fat $\mathrm{FFM}=$ fatfree mass. ${ }^{*}$ Student's $t$-test.

Table 2 Regression coefficients for constructing RV equations to obtain pRV (l) of the validation group.

\begin{tabular}{lcc}
\hline Prediction equation $^{*}$ & $\begin{array}{c}R^{2} \\
(\%)\end{array}$ & $\begin{array}{c}\text { SEE } \\
(\mathrm{l})\end{array}$ \\
\hline$-2.2671+0.0222 H$ & 23 & 0.197 \\
$-3.2362+0.0242 H+0.0280 A$ & 37 & 0.179 \\
$-3.1714+0.0235 H+0.0280 A+0.0008 W$ & 37 & 0.179 \\
\hline${ }^{*} H=$ height $(\mathrm{cm}) ; A=$ age $(\mathrm{yr}) ; W=$ weight $(\mathrm{kg})$. \\
$p$-values were $<0.001$ for $H$ and $A$, and $<0.69$ for $W$.
\end{tabular}

between them. A plot of residual (mRV - pRV) against $\mathrm{mRV}$ was also applied to assess whether the residual depends on mRV. All statistical analyses were performed using PASW statistics 18.0 for Windows (SPSS). A 2-sided $p$-value of less than 0.05 was considered statistically significant.

\section{RESULTS}

Table 1 illustrates some physical characteristics of participants in the validation and the cross-validation groups. The participants in both groups had similar height, age, weight, and other physical variables.

Mean and SD of mRV $(1.18 \pm 0.23 \mathrm{l})$ in the validation group obtained from direct measurement was significantly less than that estimated from the prediction equation $(1.29 \pm 0.15 \mathrm{l})$ developed for Caucasian population by Goldman and Becklake ${ }^{13}$, confirming the notion that RV is ethnic specific. We, therefore, attempted to develop a prediction equation for our volunteers.

Prior to constructing multiple linear regression of mRV, Pearson's correlations between $\mathrm{mRV}$ and height, age, weight, respectively, were assessed revealing $r$ of $0.477,0.315$, and 0.242 , all having $p<0.01$. Independent variables were therefore entered into the linear regression model in a sequence of height, age, and weight.

To develop a prediction equation for mRV, 121 participants in the validation group were used. Table 2 shows the results of fitting three linear regression 
Table 3 Comparisons between means of RV obtained from direct measurement and from our prediction equation in validation and cross-validation groups.

\begin{tabular}{lccccc}
\hline Data group & $\mathrm{mRV}(\mathrm{l})$ & $\mathrm{pRV}(\mathrm{l})^{*}$ & $\mathrm{mRV}-\mathrm{pRV}(\mathrm{l})$ & $p$-value & $95 \% \mathrm{CI}^{\dagger}$ \\
\hline validation & $1.189 \pm 0.223$ & $1.189 \pm 0.135$ & $0.000 \pm 0.177$ & 1.00 & $-0.032,0.032$ \\
cross-validation & $1.151 \pm 0.239$ & $1.164 \pm 0.142$ & $-0.012 \pm 0.159$ & 0.63 & $-0.063,0.039$ \\
\hline
\end{tabular}

${ }^{*} \mathrm{pRV}=-3.236243+0.024226 H+0.027993 A$. ${ }^{\dagger}$ Paired $t$-test.

equations on mRV. Using only height as an independent variable (first equation), the coefficient of determination $\left(R^{2}\right)$ and the standard error of the estimate (SEE) were $23 \%$ and $0.197 \mathrm{l}$, respectively. When height and age were entered in the model (second equation), $R^{2}$ increased to $37 \%$ and SEE decreased to 0.179 l. $p$-values of regression coefficient for height and age were less than 0.001 indicating that these two variables were highly correlated to mRV. In the third equation, in which height, age, and weight were included, $R^{2}$ and SEE were $37 \%$ and 0.179 , respectively. Thus an improvement in $R^{2}$ from the second to third equation was very small and $p$-value for weight was 0.69 indicating that weight was not related to $\mathrm{mRV}$. The model proposed was, therefore, the second equation.

Internal validity of the proposed regression equation was then evaluated using a sample of 121 participants by comparing mRV and pRV. The difference between $\mathrm{mRV}$ and $\mathrm{pRV}$ had the average of 0 and SD of 0.177 , which was not statistically significant with $p=1.00$ (Table 3). The mRV and $\mathrm{pRV}$ had a good positive correlation with $r=0.605$. The scatter plot of $\mathrm{mRV}$ and pRV (Fig. 1a) revealed that their values are around the line of equality with relatively good agreement. The plot of the difference between mRV and $\mathrm{pRV}$ against $\mathrm{mRV}$ showed limit of agreement having mean \pm 1.96 SD between -0.3124 and 0.3822 (Fig. 1b). It seemed that agreement between $\mathrm{mRV}$ and pRV was highest when mRV was approximately at 1.21.

In terms of external validity, the second regression equation was cross-validated in the other set of 40 participants. The $\mathrm{mRV}$ and $\mathrm{pRV}$ were similar with the mean difference of $-0.012, \mathrm{SD}=0.159$, and $p=0.63$ (Table 3 ). The scatter plot of $\mathrm{mRV}$ and $\mathrm{pRV}$ also showed that $\mathrm{mRV}$ and $\mathrm{pRV}$ are highly correlated $(r=0.766$, Fig. 1c) indicating that the second equation was valid and can be used to predict $R V$ in another set of individuals. The plot of the difference between $m R V$ and pRV against mRV (Fig. 1d) showed very similar results to a set of 121 participants (Fig. 1b).

When the second equation was applied to a total sample of 161 volunteers, scatter plot of pRV against
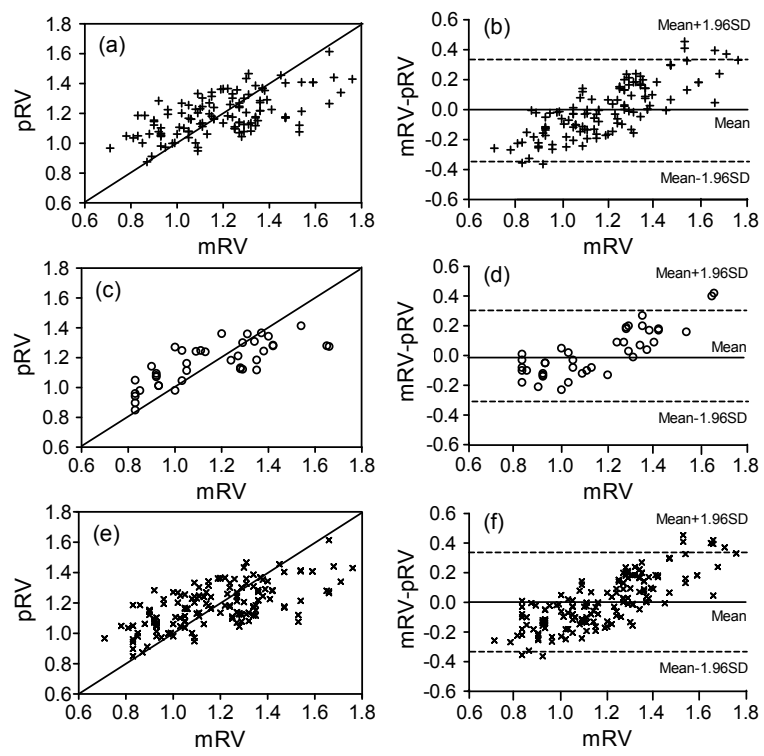

Fig. 1 The scatter plots of predicted RV (pRV) against measured RV (mRV): (a) the validation group; (c) the cross-validation group; (e) total subjects. The scatter plots of $\mathrm{mRV}-\mathrm{pRV}$ against $\mathrm{mRV}$ : (b) the validation group; (d) the cross-validation group; (f) total subjects.

mRV (Fig. 1e) and plot of their difference against $\mathrm{mRV}$ (Fig. 1f) were similar to those from 121 participants (Fig. 1a, Fig. 1b).

In view of the ethnic dependency in $R V$, it is of interest to compare our prediction equation 2 with those of other studies using 121 volunteers (Table 4 and Fig. 2). The pRV of $1.30 \pm 0.15 \mathrm{l}$ obtained from Goldman and Becklake equation ${ }^{13}$ was higher than Thai mRV which is $1.19 \pm 0.22 \mathrm{l}$ with $p<0.001$. Chin et al equation ${ }^{7}$ gave the pRV of $1.18 \pm 0.121$ that was very similar to Thai mRV with $p=0.46$. On the other hand, both Demura, Yamaji, and Kitabayashi equation $^{14}$ and Ching and Horsfall equation ${ }^{10}$ gave much lower pRV than Thai with $p<0.001$. Fig. 2 showed scatter plots of mRV from 121 participants against pRV from different equations. Predicted RV from Chin et $\mathrm{al}^{7}$ was around the line of equality which was similar to our study (Fig. 1a). The pRV from 
Table 4 Comparisons between the residual lung volume (RV) obtained by direct measurement (mRV) in the validation group $(n=121)$ and by different prediction equations.

\begin{tabular}{lrrrc}
\hline Validation group & RV $(\mathrm{l})$ & $\mathrm{mRV}-\mathrm{pRV}(\mathrm{l})$ & $p$-value* & 95\% CI of diff* \\
\hline $\mathrm{mRV}^{\dagger}$ & $1.189 \pm 0.223$ & & & \\
$\mathrm{RV}=-3.236+0.024(H / \mathrm{cm})+0.028 A^{\dagger}$ & $1.189 \pm 0.135$ & $0.000 \pm 0.177$ & 1.00 & \multicolumn{1}{c}{$0.032,0.032$} \\
$\mathrm{RV}=-3.9+0.0813(H / \mathrm{in})+0.009 A^{13}$ & $1.296 \pm 0.152$ & $-0.107 \pm 0.191$ & 0.00 & $-0.141,-0.072$ \\
$\mathrm{RV}=-2.307+0.057(H / \mathrm{in})+0.01 A+0.005(W / \mathrm{lb})^{10} \S$ & $0.843 \pm 0.112$ & $0.346 \pm 0.215$ & 0.00 & $0.307,0.384$ \\
$\mathrm{RV}=-2.968+2.78(H / \mathrm{m})+0.01 A+0.008(W / \mathrm{kg})^{7} \|$ & $1.175 \pm 0.121$ & $0.014 \pm 0.199$ & 0.46 & $-0.022,0.049$ \\
$\mathrm{RV}=-4.40778+0.03403(H / \mathrm{cm})^{14 \pi}$ & $0.894 \pm 0.163$ & $0.295 \pm 0.204$ & 0.00 & $0.259,0.332$ \\
\hline
\end{tabular}

*Paired $t$-test compared to mRV. Independent variables: $H=$ height, $A=$ age (yr), and $W=$ weight.

${ }_{\dagger}^{\dagger}$ Obtained from present study. Ethnicity: ${ }^{\dagger}$ Thai, ${ }^{\ddagger}$ Caucasian, ${ }^{\S}$ Chinese, ${ }^{\|}$Singaporean, ${ }^{\mathbb{T} J}$ Japanese.
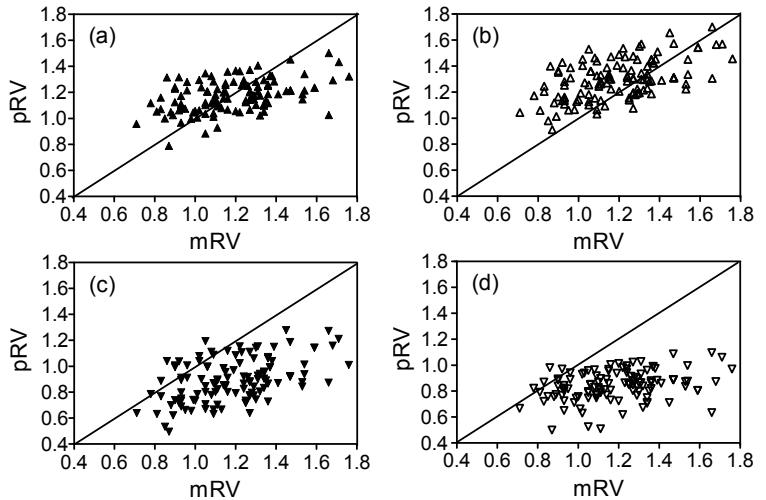

Fig. 2 The scatter plots of predicted RV (pRV): (a) Chin et $\mathrm{al}^{7}$; (b) Goldman and Becklake ${ }^{13}$; (c) Demura et $\mathrm{al}^{14}$; (d) Ching and Horsfall ${ }^{10}$, against $\mathrm{mRV}$ from the validation group.

Goldman and Becklake ${ }^{13}$ was overestimated whereas those from Ching and Horsfall ${ }^{10}$ and Demura et al ${ }^{14}$ were underestimated.

\section{DISCUSSION}

In view of the current awareness of obesity that imposes high risks for cardiovascular and metabolic diseases, an accurate measurement of body fatness is essential. Of several methods presently available to assess body composition, both the densitometry, a criterion method, and BIA, a popular field method, require an accurate RV value of a subject. This value may be obtained by direct measurements or from prediction equations. The former requires a special instrument and technical skills whereas the latter has been published by many investigators for different populations, except for the Thais. Our study is therefore the first report of a prediction equation of RV for the Thais, specifically young adult women. This population was selected for the present study because they are the target group in the prevention of obesity and the consequent diseases, and their health awareness especially obesity are very high; therefore, good co-operations and compliances of the participants are expected. Indeed, no volunteer abstained from this study.

Results in the present study showed that mRV of young adult Thai women, $1.189 \pm 0.22$ l, was significantly less than that predicted by Goldman and Becklake equation ${ }^{13}$ for Caucasians, supporting the notion that lung functions are population specific ${ }^{15}$ and that non-white populations in Asia-Pacific countries have lower lung volumes than the comparable Caucasians ${ }^{16}$. In addition, the prediction equation of RV for adult Thai women is primarily a function of height and age as in many other previously reported equations. When we compared with other prediction equations for Asian populations, however, the $\mathrm{mRV}$ of our participants differed from the values predicted from the equations for Chinese and Japanese populations, but it was comparable to that of the Chinese descendent Singaporean group ${ }^{7}$. The results further indicate that there are ethnic-differences in RV even among the Asians. The mechanisms responsible for the ethnic dependence of lung volumes and functions are not completely understood at present. Furthermore, studies in the Asian populations are scarce. It has been suggested that chest size is closely associated with lung volumes ${ }^{17}$. A study in whites and Indian descendants in England however failed to support this hypothesis ${ }^{18}$. On the other hand, Yap et $\mathrm{al}^{6}$, who studied different ethnic descendants of the Singaporean population (Malays, Chinese, and Indians), found that the differences in lung volumes could be partly explained by ethnic differences in the upper body segments. Beside anthropometric variables, other factors such as nutrition, physical activity, and environmental conditions may be contributing factors ${ }^{16}$. In support of this possible explanation, Fulambarker et al $^{19}$ recently showed that the USborn Asian Indians have higher lung functions than 
the comparable immigrant counterparts. Further, it has been reported that Cantonese ${ }^{10}$ and Taiwanese Chinese $^{8}$, but not Singaporean Chinese ${ }^{9}$, have similar lung volumes. Results in the present study that reveal similar RV to that of the Singaporean Chinese also corroborate this notion. Further investigations are however required to completely understand the confounding factors behind the ethnic dependence of lung volumes and pulmonary functions.

It should be noted that the prediction equation obtained in this study is independent of the weights of subjects. However, it does not mean that the equation is also applicable to obese individuals of the same age because the majority of our volunteers for derivation of the equation are classified as normal percentage of BF $(24.5 \pm 5 \%)$. The equation, therefore, can be applied for only a selected group (non-obese) of young women. Previously, it has been shown that the prediction equations for RV of overweight Americans (BF $>25 \%$ for men, and $\mathrm{BF}>30 \%$ for women) were different from those of normal weight men and women ${ }^{20}$. Further studies are, therefore, required to formulate the prediction equations for RV of the Asian populations.

Declarations: This work is a part of the MSc thesis of Miss Arpalak Paksaichol submitted to the School of Graduate Studies, Mahidol University.

\section{REFERENCES}

1. Going SB (1996) Densitometry. In: Roche AF, Heymsfield SB, Lohman TG (eds) Human Body Composition, Human Kinetics, Champaign, IL, pp 3-23.

2. Rossiter CE, Weill H (1974) Ethnic differences in lung function: evidence for proportional differences. Int $J$ Epidemiol 3, 55-61.

3. Schoenberg JB, Beck GJ, Bouhuys A (1978) Growth and decay of pulmonary function in healthy blacks and whites. Respir Physiol 33, 367-93.

4. Jacobs DR Jr, Nelson ET, Dontas AS, Keller J, Slattery ML, Higgins M (1992) Are race and sex differences in lung function explained by frame size? The CARDIA study. Am Rev Respir Dis 146, 644-9.

5. Korotzer B, Ong S, Hansen JE (2000) Ethnic differences in pulmonary function in healthy nonsmoking Asian-Americans and European-Americans. Am J Respir Crit Care Med 161, 1101-8.

6. Yap WS, Chan CC, Chan SP (2001) Ethnic differences in anthropometry among adult Singaporean Chinese, Malays and Indians, and their effects on lung volumes. Respir Med 95, 297-304.

7. Chin NK, Ng TP, Hui KP, Tan WC (1997) Population based standards for pulmonary function in non-smoking adults in Singapore. Respirology 2, 143-9.
8. Wu MC, Yang SP (1962) Pulmonary function study in healthy Chinese. I. Lung volume and its subdivisions. J Formos Med Assoc 61, 110-29.

9. Chuan PS, Chia M (1969) Respiratory function tests in normal adult Chinese in Singapore. Singapore Med J 10, 265-71.

10. Ching B, Horsfall PAL (1977) Lung volumes in normal Cantonese subjects: preliminary studies. Thorax 32, $352-5$.

11. Gordon CC, Chumlea WC, Roche AF (1988) Stature, recumbent length, and weight. In: Lohman TG, Roche AF, Martorell R (eds) Anthropometric Standardization Reference Manual, Human Kinetics, Champaign, IL, pp 3-8.

12. Miller MR, Hankinson J, Brusasco V, Burgos F, Casaburi R, Coates A, Crapo R, Enright P, et al (2005) Standardisation of spirometry. Eur Respir J 26, 319-38.

13. Goldman HI, Becklake MR (1959) Respiratory function tests: Normal values at medium altitudes and the prediction of normal results. Am Rev Tubercul 79, 457-67.

14. Demura S, Yamaji S, Kitabayashi T (2006) Residual volume on land and when immersed in water: Effect on percent body fat. $J$ Sports Sci 24, 825-33.

15. Yang TS, Peat JK, Keena V, Donnelly PM, Unger W, Woolcock AJ (1991) A review of the racial differences in the lung function of normal Caucasian, Chinese and Indian subjects. Eur Respir J 4, 872-80.

16. Ip MSM (2011) Lung function testing in health and disease: Issues pertaining to Asia-Pacific populations. Respirology 16, 190-7.

17. Donnelly PM, Yang TS, Peat JK, Woolcock AJ (1991) What factors explain racial differences in lung volumes? Eur Respir J 4, 829-38.

18. Whittaker AL, Sutton AJ, Beardsmore CS (2005) Are ethnic differences in lung function explained by chest size? Arch Dis Child Fetal Neonatal Ed 90, F423-8.

19. Fulambarker A, Copur AS, Cohen ME, Patel M, Gill S, Schultz ST, Quanjer PH (2010) Comparison of pulmonary function in immigrant vs US-born Asian Indians. Chest 137, 1398-404.

20. Miller WC, Swensen T, Wallace JP (1998) Derivation of prediction equations for RV in overweight men and women. Med Sci Sports Exerc 30, 322-7. 